\section{§31. Turbulence Theories and Modelling of Fluids and Plasmas}

Yoshizawa, A., Yokoi, N. (Univ. Tokyo), Itoh, S.-I. (Kyushu U.), Itoh, K.

The theoretical study of turbulence in electrically nonconducting fluids has undergone a development very different from its counterpart of plasma turbulence. In fluid flows, the growth of disturbances imposed on the laminar state of each flow is investigated on the basis of linear and nonlinear stability analyses. In the case that the laminar state is unstable, it is not rare that initial small disturbances rapidly evolve into a fully-developed turbulent state. The fully developed state of turbulence is sustained by the continuous supply of energy, for instance, through the imposition of pressure. In this situation, the fully developed state of turbulence may be studied in distinct separation from the initial stage of growing disturbances.

In the history of the theoretical study of turbulence, much attention has been paid to homogeneous, isotropic turbulence free from a spatially-varying mean flow. Especially, the famous $-5 / 3$ power law for the energy spectrum, which is derived with the aid of the concept of the inertial range, is a primary target of the theoretical study of turbulence, greatly contributing to the formulation of statistical theoretical approaches. A systematic approach to the study of inhomogeneous turbulence is indispensable for improving the current turbulence models and constructing new ones. This situation has stimulated the study of inhomogeneous-turbulence theory.

In contrast to fluid turbulence, the fullydeveloped state of plasma turbulence is not always a matter of theoretical concern, specifically in fusion plasma, for such a state is what is to be avoided for efficient plasma confinement. Then the primary target of the theoretical study of plasma turbulence is the stage of instability growth and the subsequent highly nonlinear regime subject to strong inhomogeneity. Turbulence in plasmas has several characteristic features. One is that the fluctuation level becomes high through instabilities driven due to the inhomogeneity. Inhomogeneities exist for plasma parameters (e.g., density and temperature) as well as for the fields (e.g., magnetic field and radial electric field). These inhomogeneities couple so as to drive and/or suppress instabilities and turbulent fluctuations. Fluctuations often have a very long correlation length along the magnetic field line and are quasi-two-dimensional. In addition, the mobilities of electrons and ions differ prominently. The inhomogeneities, the anisotropy due to the strong magnetic field, and the difference of ion and electron mobilities have strong influences on the linear properties of plasma waves as well as on plasma turbulence. In many cases, instabilities develop into strong turbulence, so that the decorrelation rate caused by the nonlinear interactions is usually of the same order of or much larger than the damping rate (growth rate) of the linear eigenmode. Theoretical methods developed for fluid turbulence are helpful for the study of these phenomena.

In the quest of understanding anomalous transport in confined plasmas, investigation of turbulent fluctuations has been a central theme. In particular, after finding the $\mathrm{H}$-mode in ASDEX tokamak, it has been widely recognized that the plasma profile could have a variety of forms, and changes between them could occur as sudden transitions. One of the keys to understanding the structural formation and transitions in plasmas is the study of mutual interactions of the plasma inhomogeneity, electric-field structure, and fluctuations. This theoretical progress is based upon the development of turbulence theories in fluids and plasmas. It is necessary and timely to survey the basis and recent development of turbulence theories in order to establish a perspective for future research on turbulence, turbulent transport, and structure formation.

In this review article [1], we describe the common and different characteristics between fluid and plasma turbulence. The article is organized as follows. In $\S 2$, we give the fundamental equations for electrically nonconducting and conducting fluids and plasmas. In $\S 3$, we give a review of the theoretical approaches to homogeneous turbulence and the phenomenological methods for inhomogeneous turbulence, that is, turbulence modelling. An inhomogeneous turbulence theory is explained in relation to the theoretical derivation of turbulence models. The approach to electrically nonconducting fluids is extended to magnetohydrodynamic flow, and the turbulent dynamo is discussed in the context of astrophysical and fusion plasmas. In $\S 4$, various theoretical approaches to plasma turbulence are illustrated with examples of applications. Emphasis is put on how the theory of turbulence is applied to systems composed of components of different mobility that have strong inhomogeneity and anisotropy. The connection with the current fluid turbulence theories is also sought in order to shed light on the similarity and difference between fluid and plasma turbulence. Summary is presented in $\$ 5$.

\section{Reference}

[1] A. Yoshizawa, S-I. Itoh, K. Itoh, N. Yokoi: Plasma Phys. Contr. Fusion 43 (2001) R1 